\title{
Second-order multiple-quanta flux entry into a perforated spherical mesoscopic superconductor
}

\author{
Ben Xu, M. V. Milošević, and F. M. Peeters* \\ Departement Fysica, Universiteit Antwerpen, Groenenborgerlaan 171, B-2020 Antwerpen, Belgium \\ (Received 2 August 2010; revised manuscript received 25 October 2010; published 1 December 2010)
}

\begin{abstract}
Flux entry in type-II superconductors without prominent symmetry is a first-order phase transition, where flux enters conventionally gradual in units of a flux quantum. Here we show that neither is necessarily the case in a mesoscopic superconducting sphere with a perforation. In axially applied magnetic field, vortices initially occupy the hole, and can oppose further flux entry in the sample. As a result, multiple-quanta flux entry is found at significantly higher field, and it can manifest as a second-order transition due to suppressed geometric barrier at the equatorial belt of the sample. At high fields a new state is found, with gradually destroyed condensate from the equator inwards, the exact opposite of surface superconductivity.
\end{abstract}

DOI: 10.1103/PhysRevB.82.214501

PACS number(s): 74.78.Na, 74.25.Op, 74.25.Ha

\section{INTRODUCTION}

Being nanoscale and fully three-dimensional (3D) objects, susceptible to magnetic interactions ${ }^{1-4}$ but also driven by electric current, ${ }^{5}$ having magnetic-field and temperaturedependent properties, ${ }^{6}$ and hosting both Cooper pairs and normal electrons, ${ }^{7}$ vortices in superconductors are one of the most prominent study objects of the last decade. The rising interest in vortices came along the interest in mesoscopic superconductivity, ${ }^{8-15}$ as scientists realized that many properties of superconducting samples, including vortex matter, can be tailored by the sample size and shape.

Vortex entry in superconductors is generally a first-order phase transition. In increasing magnetic field, strong Meissner currents develop in the sample to screen out the applied field. They are largest at the sample edges, where consequently the superconducting order parameter is weakest. After the critical velocity of the superconducting condensate is reached, ${ }^{16}$ the Cooper pairs break, and weak points for vortex entry are created at the sample edges. The energy associated with vortex crossing the screening currents in the sample, together with the experienced repulsion from the pre-existing vortices (if any), form together a BeanLivingston (BL) barrier, i.e., the energy barrier for vortex nucleation in the sample. ${ }^{17}$ Its presence ensures the existence of hysteresis of flux entry and exit in mesoscopic superconductors as a function of the applied magnetic field.

Thus the vortex enters the sample through a weak point, i.e., the surface area that can be as small as a single point in which the critical conditions are met. This is where the three dimensionality of the vortex line becomes important since it has to overcome the entire edge of the sample parallel to the magnetic field. As a consequence, the vortex enters the sample gradually, as a function of critical conditions changing in the neighborhood with, e.g., further increasing field, and depending on its own elasticity. Finally, the entire vortex enters the sample, but it exceeds its minimal needed length (the sample thickness), and this is energetically costly. This additional contribution to the energy barrier is named the geometric barrier, as it stems from the sample shape.

Such a geometrical barrier can be therefore successfully suppressed in samples with oblate edges, i.e., with spherical or toroidal shape. Nevertheless, vortex entry remains a first- order transition, unless the action of the Meissner currents driving the entering vortex toward the center of the sample is fully counteracted by another opposing force, so that the Bean-Livingston barrier vanishes. In what follows, we will show that this is possible in a spherical superconductor with a central perforation. The force opposing the vortex entry will be the repulsion by the previously trapped vortices in the hole. The balance of the competing forces will therefore depend on the applied magnetic field, the size of the sample, the size of the hole, and the vorticity but also the number of newly entering vortices. As a consequence, some unconventional vortex transitions for mesoscopic superconductors can be expected.

Our sample is a sphere of radius $R$, with axial and cylindrical perforation with radius $R_{h}$. In the past, a number of works dealt with the properties of a superconducting disk with a hole and rings in general, ${ }^{18,19}$ as well as hollow cylinders. ${ }^{20}$ The common conclusion was that vortices favorably reside inside the hole, and that such vortex trapping leads to an increased upper critical field of the sample. However, the number of vortices that fit inside a hole depends on the hole itself (its shape and size) $)^{21}$ but also on the sample geometry, i.e., the proximity of another hole ${ }^{22}$ or the proximity of the sample edges. In Fig. 1, we show the obtained maximal number of vortices in a hole (saturation vorticity $n_{s}$ ) as a function of the hole radius and the sample radius, and that for a cylinder and a sphere of equal volume. The difference between results for two geometries is astounding, and this is the core objective of this study.

\section{THEORETICAL FORMALISM}

The results are obtained and checked using two variants of the Ginzburg-Landau (GL) formalism, for an extreme type-II superconductor. In one approach, we solve numerically the nonlinear GL equation for the order parameter $\psi$,

$$
(-i \vec{\nabla}-\overrightarrow{\mathbf{A}})^{2} \psi=\left(1-|\psi|^{2}\right) \psi,
$$

in applied vector potential $\overrightarrow{\mathbf{A}}=(H y / 2,-H x / 2,0)$ from a magnetic field $H$ applied axially to the sample, and with 

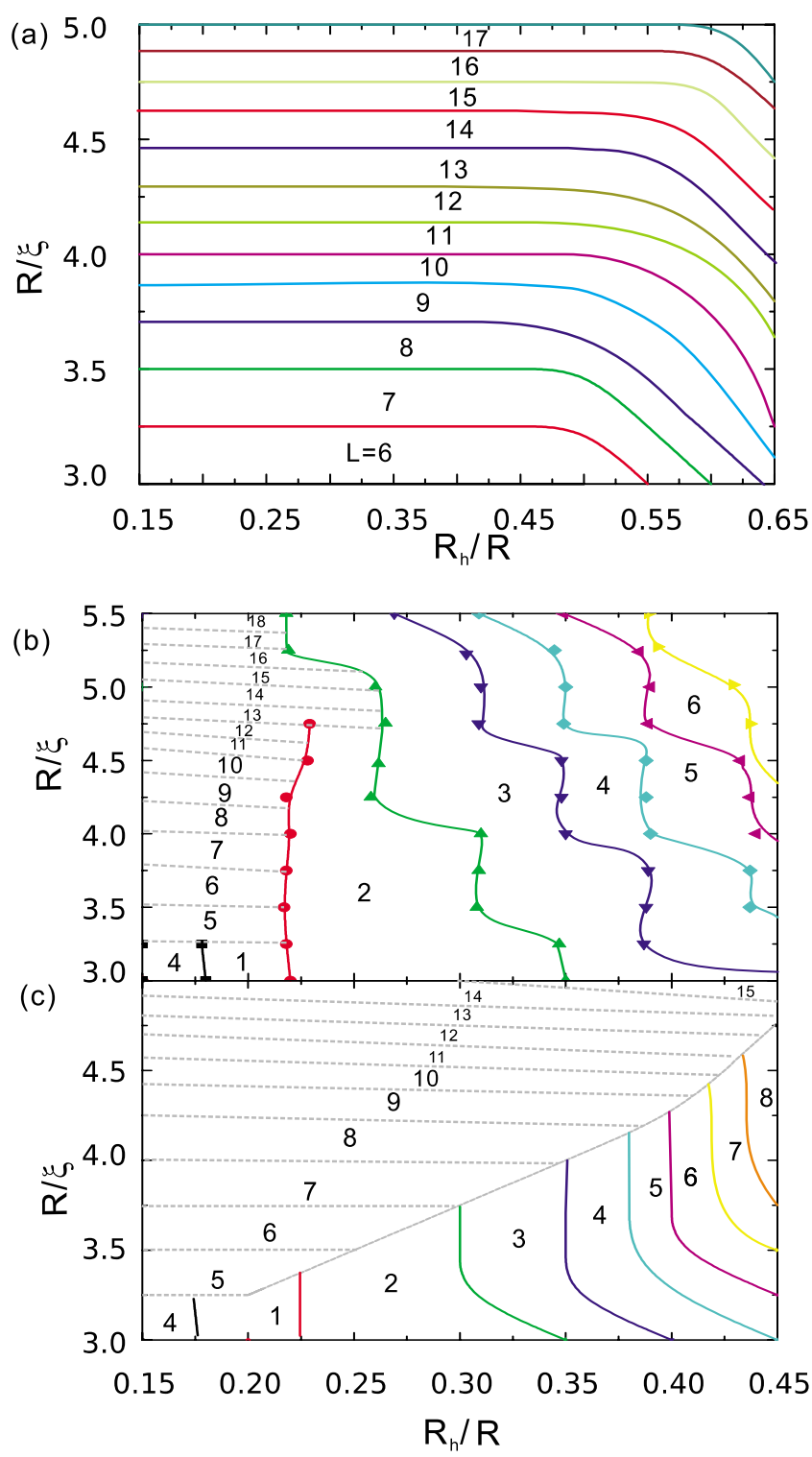

FIG. 1. (Color online) The saturation vorticity of the hole with radius $R_{h}$, in (a) a cylinder and (b) a sphere of radius $R$ (and of same volume as the cylinder). (c) The maximal possible vorticity of the sample in (b).

Neumann boundary conditions applied at the sample surfaces. Solution of Eq. (1) minimizes the free energy of a sample with volume $V: \mathcal{F} / \mathcal{F}_{0}=\frac{1}{V} \int|\psi|^{4} d V$, where $\mathcal{F}_{0}$ is the condensation energy $H_{c}^{2} / 4 \pi$. In above equations, all distances are expressed in units of the coherence length $\xi(T)$, the vector potential in $\phi_{0} / 2 \pi \xi_{0}$, and the order parameter in $\sqrt{-\alpha / \beta}$ with $\alpha, \beta$ being the GL coefficients. For details of the approach, we refer to Ref. 6.

The second approach is based on the expansion of the order parameter, ${ }^{23} \quad \Psi=\sum_{k=1}^{N} C_{k} \psi_{k}$ in the orthonormal eigenfunctions of the $3 \mathrm{D}$ kinetic-energy operator $\left(i \hbar \vec{\nabla}_{3 \mathrm{D}}-e^{*} \overrightarrow{\mathbf{A}} / c\right)^{2} \psi_{k} / 2 m^{*}=\epsilon_{k} \psi_{k}$. In cylindrically symmetric structures, these eigenfunctions have the form $\psi_{k=(L, n)}=\exp (i L \phi) f_{n}(r, \theta)$, where $L$ is the angular momentum, $\phi$ is the azimuthal angle around the sample axis, and the index $n$ counts different states with the same $L$ starting from the lowest-energy one. The eigenfunctions $f_{n}$ are real and their corresponding eigenvalues are obtained numerically for each $L$, with Neumann boundary condition applied. The typical number of considered eigenfunctions is $N=10-50$, with $L \in(0,10)$ and $n \in(1,2)$. Finally, the energy minimization with respect to the complex variables $C_{k}$ leads to the orderparameter profile of the stable vortex states.

\section{NOVEL VORTEX TRANSITIONS}

Returning to the results, the data shown in Fig. 1(a) demonstrate classic mesoscopic vortex behavior. In a cylinder with small radius, the vortices are compressed laterally into a giant vortex ${ }^{24-26}$ and the maximal vorticity increases one by one as the sample is made larger. Small perforation placed in the center of the cylinder has very little effect, as confinement dominates and a giant vortex is formed in the center of the sample regardless of the size of the hole. However, starting from the hole size of approximately $R_{h}=1 \xi$, the perforation is able to trap vortices, with the maximal occupation number (saturation number) of the hole proportional to the hole size. The hole also starts to affect the upper critical field of the sample, which leads to an increase in the maximal vorticity of the sample, as shown in Fig. 1(a). There the vortex saturation number of the hole $n_{s}$ cannot be distinguished from the maximal vorticity, since the vortex state at high applied field is always a giant vortex, with the vortex core always located in the center of the sample, i.e., inside the hole.

Similar behavior can be found in perforated spheres, however only when the perforation is very small. Beyond $R_{h}=0.175 R$, the saturation vortex number of the hole becomes independent of the maximal possible vorticity in the sample. It still increases as the hole is made larger but also has a very strong effect on the final vortex state at the upper critical field. This is illustrated in Fig. 1(c), where we show that for geometrical parameters of the cylinder that could host up to 11 vortices $\left(R=4.1 \xi, R_{h}=1 \xi\right)$, the corresponding sphere has a maximal possible vorticity of just 8 and that is reached at an intermediate magnetic field. Close to the critical field, the vorticity actually drops to just 2 , with both vortices trapped inside the hole.

To understand the above astonishing differences in flux entry in samples of different 3D geometry, we show in Fig. 2 the calculated free-energy landscape for a perforated (a) cylinder and (b) sphere, as a function of the applied magnetic field. Both samples are of equal radius $R=4.0 \xi$ and with the same radius of the hole $R_{h}=1.4 \xi$. As expected, vortices penetrate sequentially in the cylindrical sample in increasing magnetic field, and the maximal vorticity is 12 . On the other hand, the vorticity $L$ of the spherical sample increases gradually up to $L=3$ in an applied field of $H=0.75 H_{c 2}$. Surprisingly, no new energy levels are found in a large range of magnetic field, and the $L=3$ energy curve stretches up to a high magnetic field of $H=1.95 H_{c 2}$. However, the superconducting state does change along this energy line- the vorticity actually changes through a second-order transition from $L=3$ to $L=7$, before the final first-order transition to the $L=4$ state as the field is increased. Thus in the ground-state 


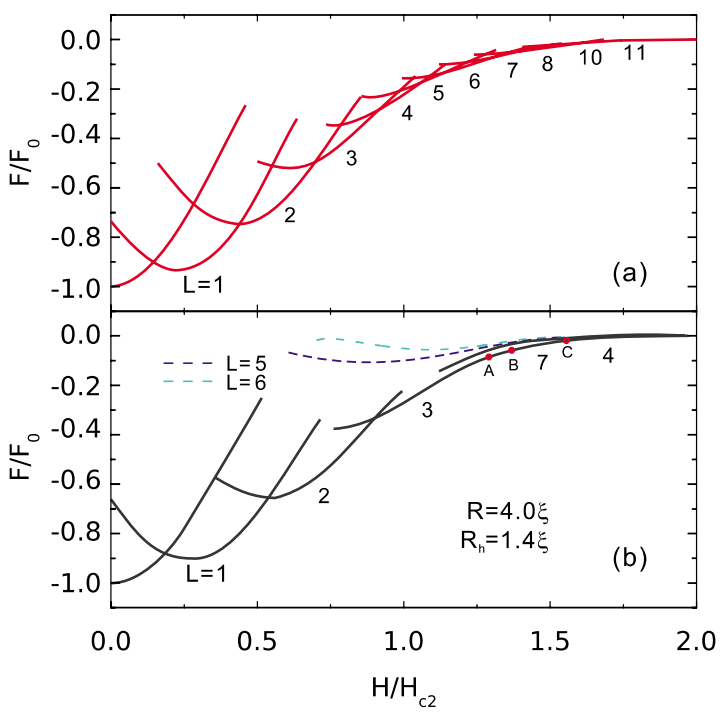

FIG. 2. (Color online) The free-energy landscape and stable vortex states as a function of the axially applied magnetic field, in (a) a perforated cylinder, compared to (b) a superconducting sphere with a hole. In (b), the dashed curves show the higher energy of $L=5$ and $L=6$ states, which were not found stable but were artificially constructed by the expansion method.

three fluxes are expelled with increasing magnetic field. Characteristic states, labeled A, B, and C in Fig. 2, are shown in Fig. 3(a) as the Cooper-pair density contourplots and the contourplots of the phase of the order parameter in the equatorial plane of the sample. States A and B show the gradual and simultaneous entry of four vortices in the sample in the

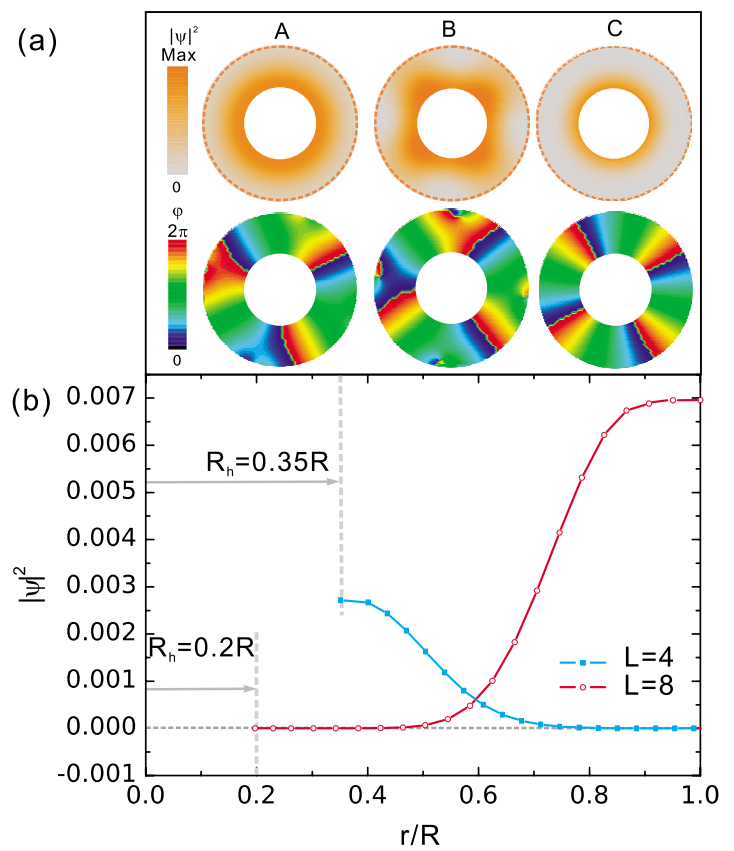

FIG. 3. (Color online) (a) The Cooper-pair density and phase contourplots of the $L=3, L=7$, and $L=4$ states, labeled as A, B, and C, respectively in Fig. 2. (b) The radial Cooper-pair density profile prior to destruction of superconductivity in two spheres with radius $4.0 \xi$ but with different radii of the holes (cf. Fig. 1).
$L=3$ state, forming the $L=7$ state, and state $\mathrm{C}$ is the last surviving superconducting state in increased magnetic field for this geometry. There we note the particularly weak order parameter at the sample edges in the central plane. In Fig. 3 (b), the radial Cooper-pair density profile prior to destruction of superconductivity is plotted for two spheres with the same radius $R=4.0 \xi$ but with different radius of the hole, $R_{h}=0.2 R$ and $R_{h}=0.35 R$. The former corresponds to the giant-vortex formation in the sample, with maximal vorticity equal 8. On the other hand, the latter is the case of Fig. 2, where four vortices are trapped by the hole and no other vortex exist in the sample. Instead, superconductivity is utterly destroyed on the equatorial belt, where the stray magnetic field is largest. The existence of this equatorial normalstate belt is phenomenologically exactly opposite to the case of surface superconductivity, and yet unreported in the field of mesoscopic superconductivity. Beside this, the found second-order multiple-quanta $L=3 \rightarrow 7$ transition is also surprising and unique. In what follows, we analyze these phenomena in greater detail.

The key in the analysis of the above phenomena is the geometry of the sample. Namely, in a spherical sample the geometric barrier is virtually absent, and vortices can nucleate as points on the equator, and grow in length as they approach the center of the sample in increasing magnetic field. However, further vortex entry becomes nontrivial since the preexisting vortices repel the new ones. This is still not sufficient for the found surprising phenomena since vortices can still rearrange to an energetic consensus. ${ }^{27,28}$ In the present case however, the central perforation pins the first entering vortices, and their rearrangement upon further flux entry is not possible. This enlarges the BL barrier for the entry of new vortices. This barrier is very firm since the geometric barrier for pinned vortices to leave the hole is very large. For this reason, vortices are unable to enter the sample beyond the $L=3$ state in Fig. 2, up to the applied field sufficiently large to overcome the BL barrier, at which point new vortices enter the sample through a second-order transition since the BL barrier is exactly matched by the magnetic "pressure" from outside the sample. In this particular case, this threshold applied field is also sufficient for four additional flux quanta to enter the sample, and we find the transition to the $L=7$ state. Further increase in the applied field compresses vortices toward the sample interior, and results finally in the entry of one more vortex into the hole, which is a first-order process. To counteract the repulsion of four vortices trapped in the hole and add more vortices to the system, the field should be increased further, however such a large field induces large equatorial screening current in the sample and the superfluid velocity exceeds the depairing one. As a result, the normal-state belt is formed, being more energetically favorable than the appearance of new localized vortices in the sample.

\section{ENERGY BARRIERS AND THERMAL EXCITATIONS}

The understand the relation between the state with a zeroorder-parameter (normal) equatorial belt and the usual giant vortex state, we examine the saddle point between them, 


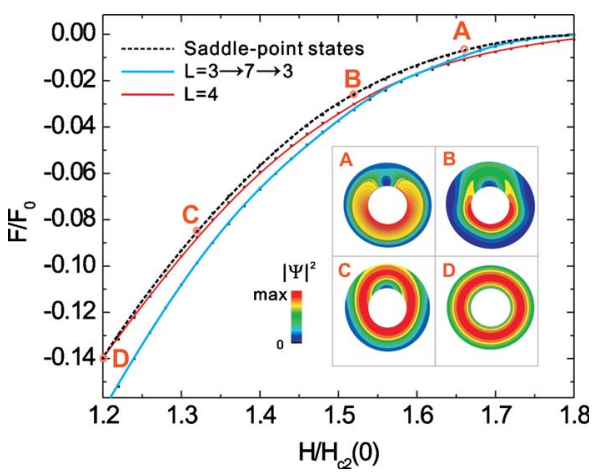

FIG. 4. (Color online) The free energy of the $L=3$ and $L=4$ states and the saddle state between them (cf. Fig. 2). In inset, the sequence of saddle points is shown through the Cooper-pair density plots at the equatorial belt of the sample, at different magnetic fields. This sequence shows the penetration of one vortex into the superconductor from outer boundary, finally residing inside the perforation.

using the expansion method from Ref. 23, as already outlined in the theoretical formalism. In Fig. 4, we show the calculated free energy, and the saddle state as transition from the normal belt with $L=3$ state to the $L=4$ state. As shown in inset of Fig. 4, one vortex forms at the normal belt (inset A), gradually moves inwards (interacting with the currents surrounding the perforation, see insets $\mathrm{B}$ and $\mathrm{C}$ ), and finally merges with the three vortices trapped inside the hole to form the giant vortex state of $L=4$. The very existence of the saddle state directly proves the first order transition between $L=3$ and $L=4$ states at high field while at the same time no saddle points can be found for the second-order transition between $L=3$ and $L=7$ states at lower fields, where $L=3$ state with the normal belt is formed from $L=7$ state by increasing magnetic field.

Using the same approach, we can now calculate the energy barrier $\Delta F$ between different vortex states and saddle points between them. This is essentially done by subtracting the energy of the metastable (higher energy) states from that of the saddle points. This is very important in the context of thermal fluctuations, which may drive the system from one equilibrium to another close to $T_{c}$, exactly where the GL theory is valid. To give a correct feel of the importance of the thermal fluctuations in the present study, we take the temperature in the calculation of $T=0.9 T_{c}$, for $T_{c}=9 \mathrm{~K}$. In that case, we can rescale the results of Fig. 2 to a sample at $T=0.9 T_{c}$ with size $R=12.6 \xi(0)$ and $R_{h}=4.42 \xi(0)$. We take $\xi(0)=10 \mathrm{~nm}$ and for $\kappa=20$, which roughly corresponds to $\mathrm{Nb}$ as superconducting material. The shape of the energy barriers between $L=1,2$, and 3 states, shown in Fig. 5(a), is generally the same as the ones described earlier in Ref. 23, being the highest at the magnetic field where two states interchange as the ground state of the system. However, the most interesting physics in the present system lies beyond the $L=3$ state, where with increasing magnetic field first the $L=7$ state is formed, and is then reduced back to the $L=3$ state with the normal belt at the equator of the sample. In this case, as shown in Fig. 5(b), the energy barrier changes with magnetic field in a somewhat different fashion, with a clear
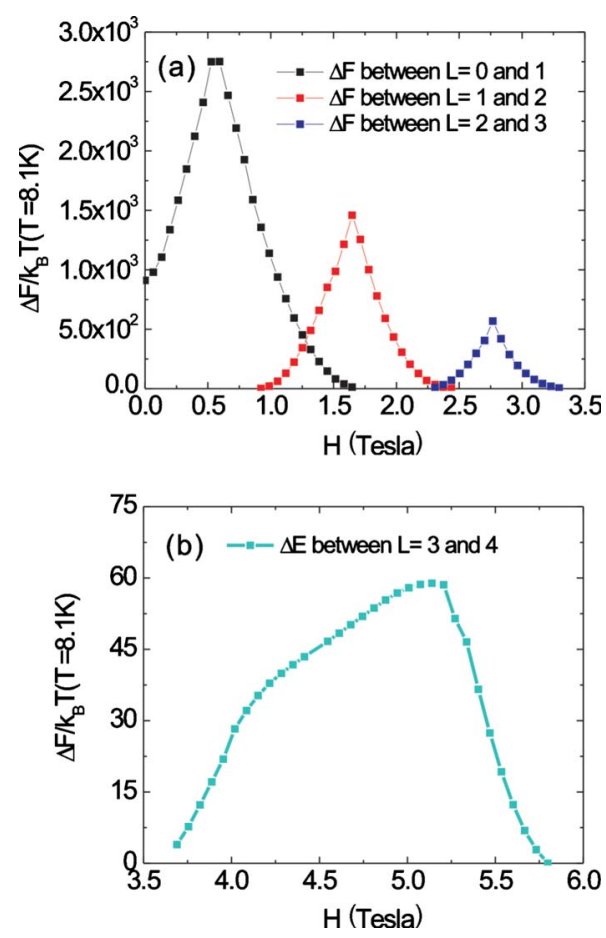

FIG. 5. (Color online) (a) The energy barriers between different vortex states from Fig. 2 (with vorticity $L \leq 3$ ), corresponding to a $\mathrm{Nb}$ sample with $R=126 \mathrm{~nm}$ and $R_{h}=44.2 \mathrm{~nm}$ at $T=8.1 \mathrm{~K}$, scaled to thermal energy $k_{B} T$. (b) The energy barrier at high fields, between the vortex state with $L=4$ and the one with $L=3$ and a normal equatorial belt.

change in $\Delta F(H)$ slope at the $L=3-7$ transition. Upon transition to the $L=4$ state in the ground state of the system, the energy barrier decreases very similarly to the states shown in Fig. 5(a). More importantly, in all curves in Fig. 5 the energy barrier is found to be much larger than the thermal energy $k_{B} T$. Note however that the barriers become comparable to the thermal energy at high magnetic fields [cf. Fig. 5(b)] so that thermal fluctuations cannot be entirely neglected. It is exactly those magnetic fields where the multiple-flux quanta entry in the system can be triggered, and thus thermal fluctuations may hide this phenomenon in experiment. Nevertheless, the latter will take place only in sufficiently large samples where the energy barriers are very low, and not in the truly mesoscopic samples where influence of the sample boundaries is extremely prominent and barriers are high. Note however that in high- $T_{c}$ samples thermal-driven transitions may take place, since there $\kappa$ is large which lowers the energy barriers, while $T_{c}$ is large and increases the thermal energy.

\section{SUPERCURRENTS AT SAMPLE BOUNDARIES}

Arguably, the strength of the barriers in a 3D superconducting geometry can be analyzed through the strength of the supercurrents, typically maximal at the outer boundaries. We therefore calculated the current in the sample using 


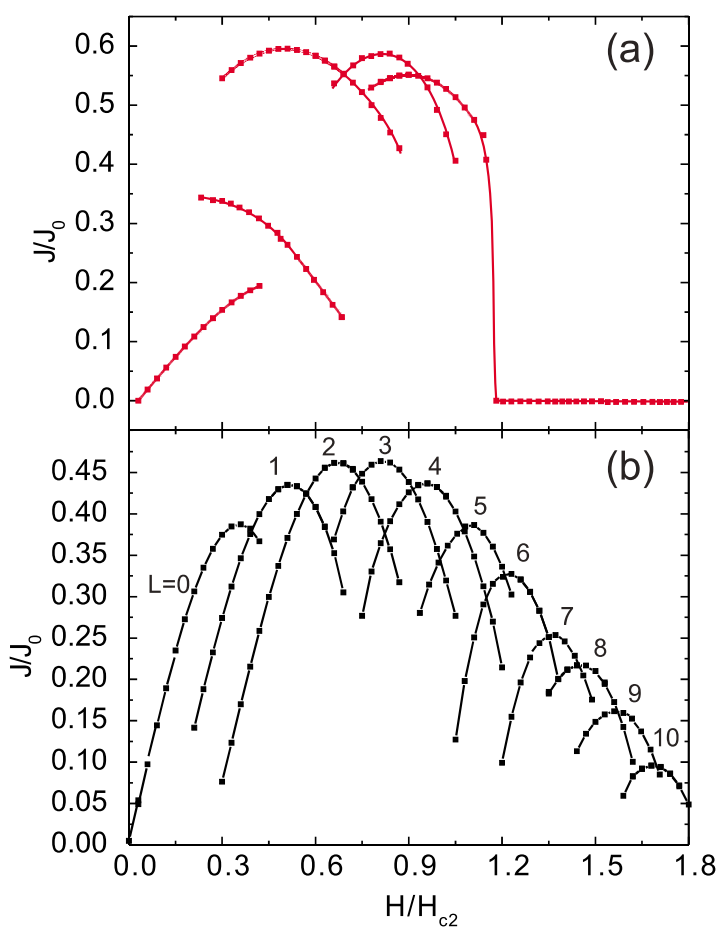

FIG. 6. (Color online) The calculated current density at the (a) inner and (b) outer boundaries of a perforated cylinder, plotted as a function of the applied magnetic field (corresponding to Fig. 2).

$$
\overrightarrow{j_{3 \mathrm{D}}}=\frac{1}{2 i}\left(\Psi^{*} \vec{\nabla}_{3 \mathrm{D}} \Psi-\Psi \vec{\nabla}_{3 \mathrm{D}} \Psi^{*}\right)+|\Psi|^{2} \vec{A}
$$

and we paid special attention to the current amplitude as a function of applied field at both inner and outer edges of the samples. In Fig. 6, we show the results for a perforated cylinder (same as in Fig. 2), where the current at the boundary (conventionally) oscillates with each vortex entry. On the other hand, upon vorticity 4 , the pinned giant-vortex outgrows the hole and the inner-edge current (and barrier) vanishes. For comparison, in Fig. 7 we show the corresponding plots for a sphere with a hole. In accordance with our findings earlier in this paper, the behavior of the currents/barriers is seemingly similar, but actually opposite. Namely, beyond $L=3$ the current and barrier at the equatorial plane diminish while remaining nonzero at the inner boundary of the sample.

\section{TUNING THE GEOMETRY AND THE INTERACTIONS}

The interaction between the inner geometric barrier of the hole and the outer BL boundary formed by trapped vortices in the hole quite obviously depends on the distance between the inner and outer surfaces of the sample, in other words on the $R_{h} / R$ ratio. We demonstrate this in Fig. 8, where we fixed the radius of the hole at $R_{h}=1.0 \xi$ and varied the radius of the sample from $R=3.6 \xi$ to $R=3.9 \xi$. As can be seen in Fig. 1(b), the saturation vorticity $n_{s}$ of the hole equals two for all the cases considered, and the maximal vorticity is always five. Nevertheless, the sequence of vortex phases and transitions between them in increased applied field are strongly affected

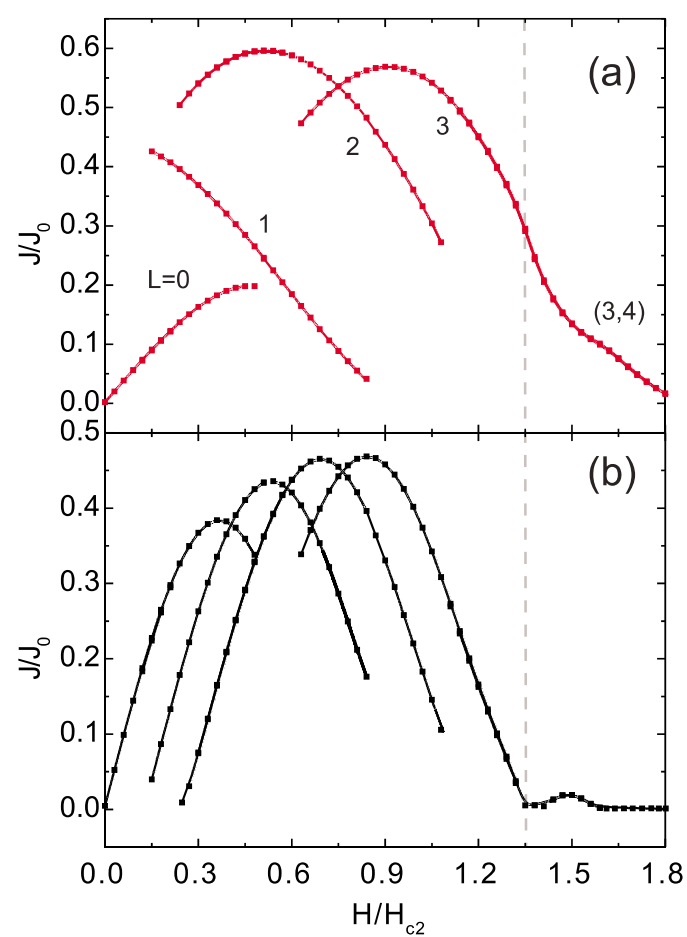

FIG. 7. (Color online) Same as Fig. 6 but for a perforated sphere. Dashed line denotes the field where the second-order transition between $L=3$ and $L=7$ sets in.

by the changing $R_{h} / R$ ratio. The equatorial contourplots of the phase of the order parameter in three samples are shown as columns in Fig. 8, with images arranged top to bottom in correspondence with increasing applied magnetic field. Both first- and second-order transitions between successive vortex states are found, indicated in Fig. 8 by open and colored arrows, respectively. In the sample with $R=3.6 \xi$, the interaction of the two vortices trapped in the hole and the outer magnetic field is strong, and we find a second-order transition and multiple-fluxon entry of three vortices simultaneously, which qualitatively corresponds to the $L=3$ to $L=7$ transition in Fig. 2. However, even in a slightly larger sample, e.g., $R=3.8 \xi$, the BL barrier is lowered at the sample edge and is no longer balanced by the repulsion from the pinned vortices. As a result, one vortex can enter the sample, which appears as a first-order transition as soon as the vortex crosses the Meissner currents at the sample edge. However, by allowing this flux entry, the system restores the balance of forces, and further flux entry from $L=3$ to $L=5$ is again of second order. Making the sample just a fraction larger, to a size of $R=3.9 \xi$, leads to first-order $L=2$ to $L=3$ and further to $L=4$ transitions, and the second-order transition is shifted to the last stage - the single vortex entry toward the $L=5$ state. In summary, by changing the size of the hole and the sample, one manipulates the interaction between inner and outer boundaries and energy barriers, and one can change any of the phase transitions between successive vortex states from first to second order, and from individual to multipleflux quanta events.

The free-energy curves in Fig. 2 clearly show the strengthening effect that the pinned vortices have on the Bean-Livingston barrier, as the vortex entry between 


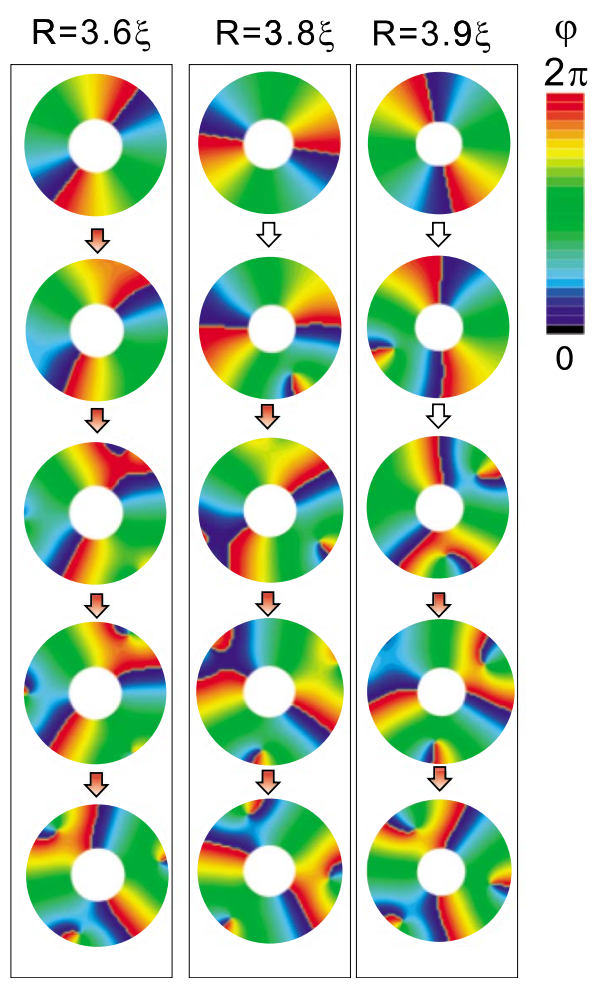

FIG. 8. (Color online) A sequence of vortex-state transitions in the ground state, with indication of the order of the phase transitions (open/colored arrows-first/second order), for different radii of the sample and fixed size of the central hole $R_{h}=1.0 \xi$. The images are arranged top to bottom in increasing applied magnetic field.

$L=0-3$ states is shifted to higher fields in samples with a weak geometric barrier. Here we discuss one more effect that the competition of the barriers has on threshold magnetic fields, in this case the upper critical field $H_{c r}$ (at which the superconductivity is destroyed). Our results are summoned in Fig. 9, for spherical samples with radii $R=3.25 \xi, 3.75 \xi$, and $4.25 \xi$, as a function of the size of the hole. Each curve exhibits a rather flat stage when the perforation is small, which corresponds to the states of weak inner geometric barrier, and the formation of giant-vortex states in the sample, up to high vorticity [such as the $L=8$ state shown in Fig. 3(b)]. This main contribution to demagnetization comes from the screening current near the outer edges, which is practically unaffected by the hole. However, a sufficiently large hole can trap a number of vortices, and in doing so effectively preserve the superconducting condensate near the inner surface of the sample while rejecting the penetration of new vortices from the outer boundary. As discussed above, this eventually leads to a gradual suppression of superconductivity from equator inwards and enhances the critical field $H_{c r}$ in a steplike manner as a function of the size of the hole. A step increase in $H_{c r}$ is found for every $R_{h} / R$ ratio for which the interplay of barriers allows one more vortex to enter the hole, and this increases the barrier for further flux entry, and therefore increases the upper critical field. These steps are clearly visible in Fig. 9(a) and are commonly found for all considered samples for $R_{h}=0.28 R, 0.35 R$, and $0.40 R$. Furthermore, in the same range of $R_{h} / R$ ratios, the upper
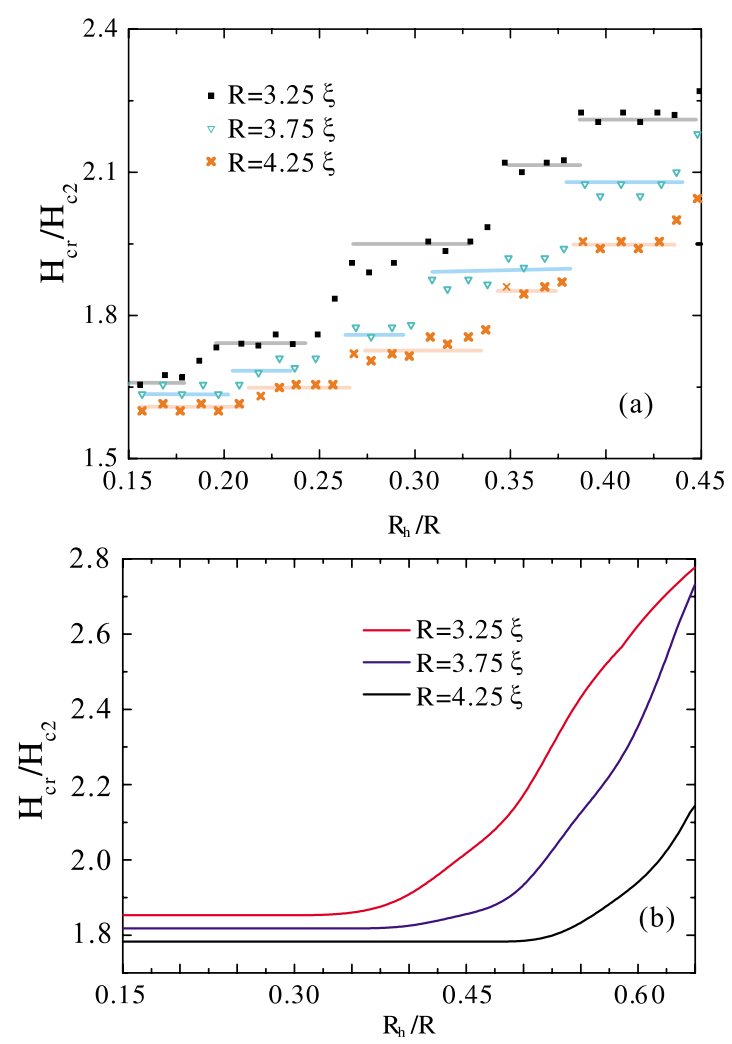

FIG. 9. (Color online) (a) The evolution of the upper critical field $H_{c r}$ of a spherical sample of radius $R$ as a function of the size of the central hole $R_{h}$, for samples of three different outer radii. (b) Idem. for a cylindrical sample of the same volume.

critical field of the sample is enhanced beyond that of a corresponding cylinder with a hole, as shown in Fig. 9(b).

\section{CONCLUSIONS}

In summary, we discussed the effect of the interplay of the geometric barrier and the Bean-Livingston barrier on vortex states and transitions between them in extreme type-II superconductors. The subtle interplay of the energy barriers can be achieved by adjusting the shape and distances between different boundaries in the system, as we demonstrate on an example of a spherical sample with a central cylindrical hole. In this case, the curvature of the outer boundary minimizes the strength of the geometric barrier for vortex entry while the cylindrical shape of the hole strongly pins the existing vortices of the sample in an axially applied magnetic field. These pinned vortices affect in turn the Bean-Livingston barrier at the outer surface of the sample, and postpone further flux entry in the sample. As a consequence, a series of sequential first- and second-order vortex phase transitions is realized, some of which can comprise several flux quanta entering the sample simultaneously, which is all very different from conventional behavior of mesoscopic superconductors, e.g., cylinders (as shown for comparison). Finally, the lack of the outer geometrical barrier in these samples can lead to the formation of a unique state at high magnetic fields, where nucleation of vortices is no longer favorable 
and superconductivity is gradually suppressed from the equatorial belt of the sample inwards, i.e., the exact opposite scenario to surface superconductivity. Based on this, even more complex behavior of the superconducting condensate is envisaged in samples with more complicated geometries, such as the 3D mesoscopic crystals recently made by electrochemistry. ${ }^{29}$

\section{ACKNOWLEDGMENTS}

This work was supported by the Flemish Science Foundation (FWO-Vl), the Belgian Science Policy (IAP), and the ESF "Nanoscience and Engineering in Superconductivity" program. *francois.peeters@ua.ac.be

${ }^{1}$ E. W. J. Straver, J. E. Hoffman, O. M. Auslaender, D. Rugar, and K. A. Moler, Appl. Phys. Lett. 93, 172514 (2008).

${ }^{2}$ S. Kang, A. Goyal, J. Li, A. A. Gapud, P. M. Martin, L. Heatherly, J. R. Thompson, D. K. Christen, F. A. List, M. Paranthaman, and D. F. Lee, Science 311, 1911 (2006).

${ }^{3}$ T. Haugan, P. N. Barnes, R. Wheeler, F. Meisenkothen, and M. Sumption, Nature (London) 430, 867 (2004).

${ }^{4}$ O. M. Auslaender, L. Luan, E. W. J. Straver, J. E. Hoffman, N. C. Koshnick, E. Zeldov, D. A. Bonn, R. Liang, W. N. Hardy, and K. A. Moler, Nat. Phys. 5, 35 (2009).

${ }^{5}$ M. V. Milošević, A. Kanda, S. Hatsumi, F. M. Peeters, and Y. Ootuka, Phys. Rev. Lett. 103, 217003 (2009).

${ }^{6}$ A. K. Geim, S. V. Dubonos, I. V. Grigorieva, K. S. Novoselov, F. M. Peeters, and V. A. Schweigert, Nature (London) 407, 55 (2000).

${ }^{7}$ A. S. Mel'nikov and V. M. Vinokur, Nature (London) 415, 60 (2002).

${ }^{8}$ L. F. Chibotaru, A. Ceulemans, V. Bruyndoncx, and V. V. Moshchalkov, Nature (London) 408, 833 (2000).

${ }^{9}$ L. R. E. Cabral, B. J. Baelus, and F. M. Peeters, Phys. Rev. B 70, 144523 (2004).

${ }^{10}$ B. J. Baelus, A. Kanda, N. Shimizu, K. Tadano, Y. Ootuka, K. Kadowaki, and F. M. Peeters, Phys. Rev. B 73, 024514 (2006).

${ }^{11}$ B. J. Baelus, A. Kanda, F. M. Peeters, Y. Ootuka, and K. Kadowaki, Phys. Rev. B 71, 140502 (2005).

${ }^{12}$ R. Geurts, M. V. Milošević, and F. M. Peeters, Phys. Rev. Lett. 97, 137002 (2006).

${ }^{13}$ M. V. Milošević, G. R. Berdiyorov, and F. M. Peeters, Appl. Phys. Lett. 91, 212501 (2007).

${ }^{14}$ J. Bonča and V. V. Kabanov, Phys. Rev. B 65, 012509 (2001).
${ }^{15}$ G. R. Berdiyorov, M. V. Milošević, and F. M. Peeters, Phys. Rev. B 76, 134508 (2007).

${ }^{16}$ M. Tinkham, Introduction to Superconductivity (McGraw-Hill, New York, 1975).

${ }^{17}$ C. P. Bean and J. D. Livingston, Phys. Rev. Lett. 12, 14 (1964).

${ }^{18}$ B. J. Baelus, F. M. Peeters, and V. A. Schweigert, Phys. Rev. B 61, 9734 (2000).

${ }^{19}$ G.-Q. Zha, S.-P. Zhou, B.-H. Zhu, and Y.-M. Shi, Phys. Rev. B 73, 104508 (2006).

${ }^{20}$ D. H. Douglass, Jr., Phys. Rev. 132, 513 (1963).

${ }^{21}$ G. S. Mkrtchyan and V. V. Schmidt, Sov. Phys. JETP 34, 195 (1972).

${ }^{22}$ G. R. Berdiyorov, M. V. Milošević, B. J. Baelus, and F. M. Peeters, Phys. Rev. B 70, 024508 (2004).

${ }^{23}$ V. A. Schweigert and F. M. Peeters, Phys. Rev. Lett. 83, 2409 (1999).

${ }^{24}$ A. Kanda, B. J. Baelus, F. M. Peeters, K. Kadowaki, and Y. Ootuka, Phys. Rev. Lett. 93, 257002 (2004).

${ }^{25}$ D. S. Golubović, M. V. Milošević, F. M. Peeters, and V. V. Moshchalkov, Phys. Rev. B 71, 180502(R) (2005).

${ }^{26}$ I. V. Grigorieva, W. Escoffier, V. R. Misko, B. J. Baelus, F. M. Peeters, L. Y. Vinnikov, and S. V. Dubonos, Phys. Rev. Lett. 99, 147003 (2007).

${ }^{27}$ B. Xu, M. V. Milošević, and F. M. Peeters, Phys. Rev. B 77, 144509 (2008).

${ }^{28}$ J. Tempère, V. N. Gladilin, I. F. Silvera, J. T. Devreese, and V. V. Moshchalkov, Phys. Rev. B 79, 134516 (2009).

${ }^{29}$ Z. L. Xiao, C. Y. Han, W. K. Kwok, H. H. Wang, U. Welp, J. Wang, and G. W. Crabtree, J. Am. Chem. Soc. 126, 2316 (2004). 